\title{
Chi dice ragna dice danno. Ecfrasi e ipostasi di Aracne nell'opera di Primo Levi
}

\author{
Mattia Cravero \\ Universià di Torino \\ mattia.cravero@unito.it
}

\section{Riassunto}

L'articolo analizza le citazioni ecfrastiche della Divina Commedia nell'opera di Primo Levi e dimostra la loro preponderanza nella produzione del chimico-scrittore. Due incisioni di Gustave Doré, in particolare, si ancorarono alla mente del giovane Levi quando, da piccolo, sfogliò e risfogliò l'opera dell'artista francese, fissandosi come un'icona inossidabile che raffigurava i versi di Dante. Ritornarono alla sua attenzione in età matura, sottoforma di simboliche reminiscenze, durante la sua attività di scrittore, favorendo la sua creatività letteraria e dimostrando un ascendente artistico che l'autore stesso negava di avere. L'intento del presente saggio è dunque dipanare il fil rouge della visualità nell'opera di Levi: tramite la comparazione dei passi e delle raffigurazioni della Commedia, ricostruendo la cronologia che intercorre tra questi rapporti, l'obiettivo è ricostruire l'influenza dell'arte nella vita e nella produzione letteraria del chimico-scrittore.

Parole chiave: Primo Levi; ekphrasis; Gustave Doré; Dante; Divina Commedia.

\section{Abstract. Chi dice ragna dice danno. Aracne's ékphrasis and hypostasis in Primo Levi's} work

The article analyses the ekphrastic quotations from the Divina Commedia and sheds light on their importance in Primo Levi's work. Two engravings by Gustave Doré stroke and get stuck in Levi's mind since when, in his childhood, he leafed the French artist's work over and over; in his mind, these soon became unforgettable representations of Dante's verses. Such figures came back to his attention when he was older, during his activity as a writer, and inspired him as symbolic reminiscences which confirm he was not alien to artistic impulses. Thus, this article aims at reconstructing and following the background of such depictions, and also at reconstructing the presence of art in Levi's work and life - through a comparison between texts and images, and by considering and interpreting their chronological order to demonstrate Doré's importance.

Keywords: Primo Levi; ecphrasis; Gustave Doré; Dante; Divina Commedia. 
E ci sono infine, beninteso, lacune anche più grosse, vuoti senza fondo, che sono vuoti miei, di una cultura autogestita, sbilanciata, faziosa, domenicale ed anche violentata: niente di musica, niente di figurativo, poco o niente dell'universo del sentimento. Tant'è, non potevo fingere di essere chi non sono. ${ }^{1}$

Questa citazione è estratta dalla Premessa alla Ricerca delle radici di Primo Levi ${ }^{2}$ e fa riferimento all'operazione di dénouement biografico-letterario richiestogli dall'esperimento editoriale. ${ }^{3}$ Il giudizio è netto: "niente di figurativo". Ed è anche vero: nella sua opera, influenzata maggiormente dalle sue esperienze di vita, dal suo mestiere di chimico e dalla sua formazione liceale, l'arte non ha un'importanza decisiva. ${ }^{4}$ Eppure, al contrario di quanto sosteneva lui stesso, a questa singolare e stupefacente espressione umana non fu completamente estraneo: lo conferma la stessa introduzione alla Ricerca, dove si cimenta in un'ékphrasis rivelatrice della sua forma mentis e del suo ampio bagaglio culturale. Tramite una similitudine, ${ }^{5}$ si descrive nell'atto di rovistare nella propria mente, decidendo quali autori includere o escludere dall'antologia:

A metà cammino mi sono sentito nudo, e in possesso delle opposte impressioni dell'esibizionista, che nudo ci sta bene, e del paziente sul lettino in attesa che il chirurgo gli apra la pancia; anzi, in atto di aprirmela io stesso, come Maometto nella nona bolgia e nell'illustrazione del Doré, in cui del resto il compiacimento masochistico del dannato è vistoso. ${ }^{6}$

È il richiamo ad alcuni tra i più irriverenti versi della Commedia: siamo nel ventottesimo canto, ottavo girone, nona bolgia, in un raccapricciante e cruento scempio di corpi. I dannati che Dante incontra sono i "seminator di scandalo e di scisma", 7 il cui contrappasso prevede che i loro corpi vengano smembrati per l'eternità da terribili piaghe: espieranno così le loro colpe, rei di aver provocato fratture epocali e profonde, di aver ridotto in frantumi la pace, il bene

1. RR, II, p. 9.

2. Per indicare l'opera primoleviana, uso i seguenti acronimi: $\mathrm{RR}=$ La ricerca delle radici. Antologia personale $; \mathrm{AOI}=$ Ad ora incerta $; \mathrm{AM}=$ L'altrui mestiere $; \mathrm{PS}=$ Pagine sparse 1947 1986; con la dicitura "Note ai testi", a cui segue per esteso il nome dell'opera di riferimento, rimando all'omonimo apparato filologico di Levi, P. (2016-2018); ogni riferimento è seguito dal numero del volume e dalla pagina da cui è estratto. Per le citazioni dal terzo volume, contenente invece le dichiarazioni e le interviste, il riferimento è composto da nome dell'intervistatore, titolo dell'intervista, anno, numero del volume e relative pagine.

3. Nel 1980, Giulio Bollati propone ad alcuni scrittori dell'Einaudi (Calvino, Volponi e altri, insieme a Levi) di realizzare un'antologia personale delle loro più importanti letture formative. In giugno Levi firma un contratto e invia una lettera al suo editor, che diverrà poi la prefazione al volume. Cfr. la ricostruzione di Belpoliti, in Note ai testi. La ricerca delle radici, II, p. 1785 per una più dettagliata delineazione della vicenda editoriale.

4. Lo sottolinea anche Belpoliti, 2015, p. 199, nella voce Arte del glossario che affronta i temi portanti della Ricerca delle radici.

5. Che è, come ha brillantemente osservato Elisabetta Abignente, uno "strumento utile a catturare, sollecitare o mantenere viva l'attenzione del lettore-spettatore" (Abignente, 2014, p. 185).

6. RR, II, pp. 6-7.

7. Dante, Inf. XXVIII, 35. 
e il quieto vivere del loro tempo. E tra di loro spicca proprio Maometto, aperto a metà come durante una toracotomia, che sfoggia il proprio corpo dissezionato, con tutte le interiora che ne fuoriescono, come un modello anatomico ambulante: ${ }^{8}$

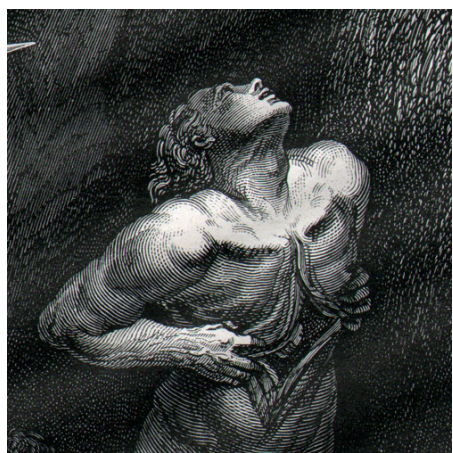

Già veggia, per mezzul perdere o lulla, com'io vidi un, così non si pertugia, rotto dal mento infin dove si trulla. Tra le gambe pendevan le minugia; a corata pareva e "l tristo sacco che merda fa di quel che si trangugia. Mentre che tutto in lui veder mattacco, guardommi, e con le man s'aperse il petto, dicendo: 'Or vedi com'io mi dilacco! vedi come storpiato è Maometto!?

Il cenno di Levi alla Commedia dantesca illustrata da Gustave Doré, di cui ebbe un esemplare in eredità dallo zio paterno,${ }^{10}$ testimonia l'importanza che un tale connubio di poesia e rappresentazioni grafiche ebbe sulla memoria del chimico-scrittore: ${ }^{11}$ la reminiscenza di questo corpus visuale funziona, in età matura, come un paradigma espressivo fondato sulla disponibilità della memoria iconica del lettore. Ricordando l'iconografia che lo aveva fortemente colpito durante l'infanzia, Levi cita un'immagine proveniente dal capolavoro figurativo di Doré, offrendo così ai suoi lettori l'opportunità di rievocare i versi della Commedia e vedere, sinesteticamente, l'emozione che traspare dietro le sue parole. Se nel Purgatorio Dante chiamava Virgilio "dolce pedagogo"12, noi potremmo dire, usando la felice definizione di Giorgio Calcagno, che Dante è a sua volta "[d]olcissimo padre"13 di Levi: un modello da cui attingere preziose immagini ormai cristallizzate all'interno di un saldo "immaginario

8. Illustrazione 55 - Inferno - Canto XXVIII, Alighieri, 1954, p. 219.

9. Dante, Inf. XXVIII, 22-31.

10. Thomson, 2002, p. 22 scrive che Levi, dopo la morte dello zio Enrico, "avrebbe ricevuto in eredità l'edizione antiquaria in tre volumi di Dante, con le timorate illustrazioni di Gustave Doré" (la traduzione italiana è mia). Nella traduzione di Eleonora Gallitelli, il sintagma che indica l'opera dantesca ("Enrico's antiquarian three-volume Dante") diventa "il raro Dante in tre volumi di Enrico" (Thomson, 2017, p. 20); questa è l'unica informazione sull'edizione posseduta da Levi. Ė probabile che l'opera in questione possa essere Dante, 1868 (e cioè la prima edizione italiana di tutta la Commedia illustrata da Doré). Verifiche ulteriori saranno possibili quando la biblioteca personale verrà aperta agli studiosi, come sostiene Bucciantini, 2016.

11. A questo proposito, Jonathan Usher ha notato che "tra i lavori che colpirono con forza la memoria poetica di Levi (e vi si ancorarono) c'è l'edizione riccamente illustrata della Divina Commedia con le incisioni di Gustave Doré" (Usher, 2004, p. 101; la traduzione italiana è mia).

12. Purg. XII, 3.

13. È il titolo di Calcagno, 2000, a cui rimando per un'analisi delle più significative citazioni e rielaborazioni dantesche nell'opera di Levi. 
reticolare". ${ }^{14} \mathrm{Ma}$, oltre alle terzine dantesche, è proprio il dispositivo creato dalla giustapposizione di testo e immagini a costituire occasione letteraria. La memoria delle incisioni che rappresentano il viaggio di Dante apre infatti nell'opera di Levi un sentiero di risonanze curioso e interessante. Uno dei casi di ékphrasis fedelmente modellata sulle illustrazioni dell'artista francese (che la critica non ha ancora analizzato né sistematizzato nel suo complesso) si riscontra infatti nell' articolo Paura dei ragni, ${ }^{15}$ confluito nell'eclettica raccolta intitolata L'altrui mestiere (1985) ma pubblicato su "La Stampa" già il 17 maggio 1981. Qui Levi torna a citare le illustrazioni della Commedia e crea un condensato di reminiscenze, descrizioni personali e ragionamenti a partire dal forte ascendente della figura di Aracne sul suo immaginario: ${ }^{16}$

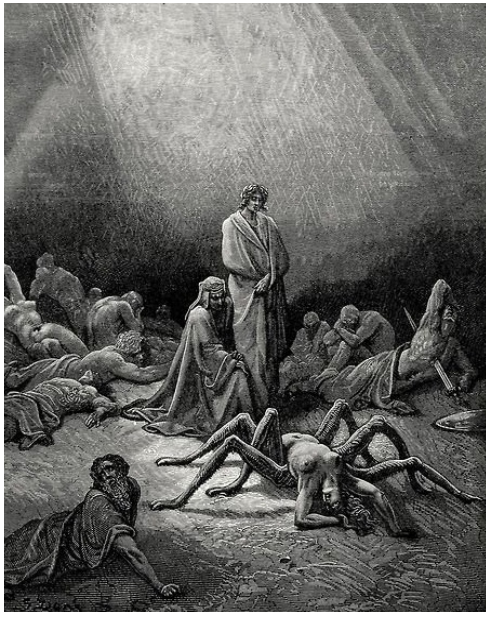

Quanto alla mia personale e tenue fobia, essa ha un atto di nascita. È l'incisione di Gustavo Doré che illustra Aracne nel canto XII del Purgatorio, con cui sono venuto a collisione da bambino. La fanciulla che aveva osato sfidare Minerva nell'arte del tessere è punita con una trasfigurazione immonda: nel disegno è "già mezza ragna", ed è genialmente rappresentata stravolta, coi seni prosperosi dove ci si aspetterebbe di vedere la schiena, e dalla schiena le sono spuntate sei zampe nodose, pelose, dolorose: sei, che con le braccia umane che si torcono disperate fanno otto. In ginocchio davanti al nuovo mostro, Dante sembra ne stia contemplando gli inguini, mezzo disgustato, mezzo voyeur. ${ }^{17}$

Si tratta di un'altra rappresentazione ecfrastica, una "rappresentazione verbale di una rappresentazione visiva", ${ }^{18}$ secondo l'efficace definizione che ne dà Massimo Fusillo scrivendo a proposito di questo fenomeno artistico nella sua

14. Mario Domenichelli, nel numero monografico di "Allegoria" dedicato alla situazione attuale della critica tematica, teorizza questo importante concetto: si tratta della rete di informazioni che si scambiano incessantemente, come tessuti neurali continuamente percorsi dagli stimoli, e si compenetrano in ogni momento, verso ogni direzione: memoria e cultura si uniscono per creare una fitta trama di rimandi che, nel suo complesso, si configura come un "reticolo che coinvolge in una miriade di possibili connessioni il rapporto tra memoria e immaginario personali e memoria e immaginario collettivi" (Domenichelli, 2008, p. 36). Ed è così anche nel caso di Levi, che riprende Dante tramite Doré, recuperando presumibilmente anche la fonte ovidiana che sta alla base dei versi danteschi.

15. Pubblicato qualche mese dopo la lettera all'editore che si sarebbe poi trasformata nell'introduzione alla Ricerca, del 2 settembre 1980 (cfr. Note ai testi. La ricerca delle radici, II, p. 1785).

16. Illustrazione 93 - Purgatorio - Canto XII, Alighieri, 1954, p. 373.

17. AM, II, p. 910.

18. Fusillo, 2012, p. 34. Rimando a questo saggio per un'analisi completa delle ibridazioni tra letteratura e arti visive con valide definizioni e interessanti proposte di metodo. 
generalità. Il commento del chimico-scrittore ha un tono avvincente, mette in risalto la simbologia della scena in cui i versi di Dante vengono rappresentati: Levi sta giocando con la loro grande significatività e la sta ritemprando grazie al potere della scrittura proprio come accade nel processo ecfrastico. D'altronde, come spiega sempre Fusillo, descrivere a parole un'opera d'arte "significa lavorare sulla dimensione visiva del linguaggio, sulla sua potenza icastica, incrinando i confini tra parola e immagine, e riempiendo la riproduzione oggettiva con proiezioni emotive" ${ }^{19}$ Le sue parole non nascondono un picco di empatia: le zampe della fanciulla che si trasforma sono, per metonimia, "dolorose", trasudano un male che solo Levi legge all'interno della raffigurazione. Da quanto scrive sembra che, contemplando l'incisione di Doré, potesse sentire lo stridio emesso dalla spasimante contrazione del suo corpo. La scena si connota soprattutto per il suo possibile significato erotico, attribuito principalmente al punto su cui si posa il curioso sguardo di Dante, che Doré rappresenta irretito, intento e attento a osservare il processo metamorfico. Anche gli erogeni "seni prosperosi" sono parte integrante della raffigurazione, di cui Levi non manca di cogliere il tono voyeuristico: diventa voyeur del voyeur e, come il Sommo, viene incantato, a metà tra curiosità e sdegno, dal processo metamorfico in atto. In realtà, nella Commedia non c'è alcuna menzione dell'anatomia di Aracne, anzi lo stesso Dante sembra pervaso da un accesso di empatia, più che disgusto: "sì vedea io te / già mezza ragna, trista in su li stracci / de l'opera che mal per te si fé". ${ }^{20}$

Al corrente della sua storia, il Sommo non poté che inserirla nella cornice dei superbi: perché la fanciulla era tanto esperta nell'arte dell'ago e del filo che nemmeno la tessitrice per eccellenza tra gli olimpici avrebbe potuto sconfiggerla. Quando poi, un giorno, la dea della saggezza vede che il mantello su cui Aracne ha trapunto i convegni amorosi degli dèi è sprovvisto di ogni benché minima imperfezione, si infuria e decide di distruggerlo, riducendo a brandelli la creazione della sua avversaria. Abbattuta dalla sconfitta e oltraggiata dalla prepotenza della dea, Aracne decide di uccidersi: vuole impiccarsi. ${ }^{21}$

L'infelice non resse e di slancio si pose in un cappio.

Pallade ne ebbe pietà nel vederla appesa e la sostenne:

'Vivi, infame' le disse, 'ma resta appesa, e l'identica

pena, affinché tu non stia in pace per il futuro,

tocchi alla tua stirpe e a tutti i tuoi discendenti! ${ }^{22}$

Nel racconto di Ovidio, fonte sia di Dante sia presumibilmente di Levi, dopo il tentato suicidio della fanciulla, Atena, mossa dalla pena per aver punito la

19. Fusillo, 2014, p. 25. Rimando a questo saggio per un inquadramento generale del rapporto letteratura-visualità nello studio delle letterature comparate.

20. Purg. XII, 43-45.

21. Così Graves, 1983, p. 54 presenta il mito, tenendo conto di tutte le sue varianti.

22. Ovidio, Met., 6, 134-138. Nella sua minuta riscrittura del mito, Levi sembra ricordarsi della lezione eziologica di Ovidio, che racconta del tentato suicidio di Aracne dopo l'ira della dea: uno dei punti focali della sua poesia su Aracne sarà proprio la sua ragnatela. 
giovane così duramente da istigarla alla morte, le risparmia il trapasso trasformandola in un ragno (anzi, in una 'ragna', per analogia alla sua arte), l'insetto che la ragazza più odiava. Così anche la corda che le toglie il respiro viene tramutata in una ragnatela: grazie alle sue nuove capacità, la ragazza può arrampicarsi e aver salva la vita, fuggendo dall'ira della dea e dando vita a una nuova specie. ${ }^{23} \mathrm{Ma}$ l'attenzione primoleviana per i ragni non si ferma qui. La figura dell'esperta tessitrice ritorna, citata letteralmente, in una poesia dove la sua presenza diventa icastica: nell'opera del chimico-scrittore gli animali sono un tratto importante e fondamentale a cui guardare, poiché assumono una valenza significativa. Rappresentano infatti, per usare l'opportuna definizione di Gordon e Belpoliti, "metafore, modelli e miti" ${ }^{24}$ grazie a cui raccontare, spesso con acume e rigore bio- ed eziologico, la varietà della vita sul nostro pianeta: gli animali sono un catalogo sconfinato di presenze reali che, agli occhi di Levi, diventano vivi oggetti di osservazione e interesse, epitomi che si prestano a una lettura dal punto di vista metaforico, le cui doti, nella prassi letteraria, possono essere traslate per creare un equivalente dei comportamenti umani. "Negli animali c'è l'enorme e il minuscolo, - diceva a Giovanni Tesio - la saggezza e la follia, la generosità e la viltà. Ognuno di loro è una metafora, un'ipostasi di tutti i vizi e di tutte le virtù degli uomini" : ${ }^{25}$ lo si vede chiaramente nella poesia del 29 ottobre 1981, anch'essa "frutto di una curiosità insoddisfatta", ${ }^{26}$ come sostenne Levi stesso, dove a parlare è Aracne, antonomastica rappresentante di tutta la sua specie, e in particolare del sesso femminile: il chimico-scrittore-poeta le dà voce, la impersona, forse interessato a combattere la sensazione di ribrezzo che provava verso la sua specie, il cui "atto di nascita" partiva proprio dall'incontro-scontro (descritto infatti come una "collisione") con l'illustrazione di Doré.

Mi tesserò un'altra tela,

Pazienza. Ho pazienza lunga e mente corta,

Otto gambe e cent'occhi,

Ma mille fiere mammelle,

E non mi piace il digiuno

E mi piacciono le mosche e i maschi.

Riposerò quattro giorni, sette,

Rintanata dentro il mio buco,

Finché mi sentirò l'addome gravido

Di buon filo vischioso lucente,

23. Proprio alla versione ovidiana del mito risulta attingere Levi, come suggerisce l'onomastica divina (si parla di Minerva, non di Atena). E peraltro, come scrive Rosati, "la fortuna di Aracne in età moderna si deve in ogni caso a Ovidio" (Ovidio, 2009, p. 243). Ho avuto modo di accertare la frequentazione delle Metamorfosi da parte di Levi lungo gli anni Settanta, definendo la presenza del testo ovidiano come una "costante citazionale" nell'opera del chimico-scrittore (sulla questione: Cravero, 2020, p. 362).

24. Belpoliti e Gordon, 2007, p. 52; la traduzione italiana è mia.

25. G. Tesio, Le occasioni? La memoria, un ponte, una ragnatela, 1984, III, p. 477.

26. G. Tesio, Le occasioni? La memoria, un ponte, una ragnatela, 1984, III, p. 477. 
E mi tesserò un'altra tela, conforme

A quella che tu passante hai lacerata,

Conforme al progetto impresso

Sul nastro minimo della mia memoria.

Mi siederò nel centro

E aspetterò che un maschio venga,

Sospettoso ma ubriaco di voglia,

A riempirmi ad un tempo

Lo stomaco e la matrice.

Feroce ed alacre, appena sia fatto buio,

Presto presto, nodo su nodo,

Mi tesserò un'altra tela. ${ }^{27}$

Levi guarda al mondo animale attraverso quello che Marco Belpoliti ha giustamente definito "cannocchiale rovesciato sul mondo umano" 28 : ne osserva i minimi particolari, dà voce al guizzo della propria fantasia e, con perizia di biologo, fantastica qui di immedesimarsi nella ragazza del mito, ritrovando in lei e nella sua specie per somiglianza o per contrasto, la stessa figura dell'essere umano, con le sue paure, vizi e inclinazioni. Aracne è un pretesto di accesso al mondo degli animali; la poesia che Levi intesse laboriosamente cela la sua grande passione per il loro mondo, ricco di piste feconde da interrogare per comprendere la lucreziana rerum natura. Interessato al loro valore simbolico, Levi li fa entrare a far parte delle sue pagine: ogni dettaglio del loro comportamento può celare un significato, un'occasione di conoscenza di uno o dell'altro aspetto più o meno nascosto del nostro mondo. Ritroviamo questo pensiero perfettamente espresso nell'articolo Lo scrittore e gli animali (poi confluito nell'Altrui mestiere sotto il nome di Romanzi dettati dai grilli) ${ }^{29}$ : è il 1976, 10 aprile, e Levi dà prova pubblica su "La Stampa" della sua capacità di guardare il mondo attorno a sé, di saper spulciare nella trama delle storie naturali che dipana per coglierne i segreti microscopici più nascosti, più curiosi. E scrive, nel suo articolo, proprio sui ragni, "inesauribile sorgente di meraviglia, di meditazioni, di stimoli e di brividi" ${ }^{30}$ Come non pensare di nuovo, leggendo queste parole, alla "collisione" con l'incisione di Doré e alla "personale e tenue fobia" di cui avrebbe scritto in Paura dei ragni? L'incontro-scontro con la 'ragna' cantata da Dante e raffigurata da Doré si rivela di fondamentale importanza: dai loro modelli, come ben dimostra la poesia succitata, Levi sceglie di concentrarsi sull'éros, non alieno alla scena riprodotta da Doré e evidente nella descrizione ecfrastica. Se si considerano da vicino i termini impiegati nella descrizione delle membra, sembra quasi di rivedere l'ombra dell'impudente e cruenta contorsione di Aracne nelle fattezze attribuitele dall'incisore francese, la stessa immagine che si impresse nella memoria di Levi, con i fuochi

27. AOI, II, p. 723.

28. Belpoliti, 2015, p. 253.

29. AM, II, pp. 851-855.

30. AM, II, p. 853. 
principali in piena evidenza: le "otto gambe", le "fiere mammelle", l'"addome gravido", la libidinosa "voglia" che ubriaca e il desiderio di "riempirmi ad un tempo / Lo stomaco e la matrice". 31

Tanto più se consideriamo bene la datazione delle opere di Levi: se la Ricerca delle radici venne messa appunto tra il giugno e il dicembre del 1980, l'edizione delle incisioni di Doré deve essere stata consultata sicuramente prima della lettera all'editore, sottoscritta il 2 settembre $1980 .{ }^{32}$ Poco tempo dopo, il 17 maggio 1981, Levi scrisse Paura dei ragni, in cui citò la raffigurazione della metamorfosi di Aracne; alcuni mesi più tardi, nell'ottobre dello stesso anno, scrisse la poesia Aracne, per verbalizzarla poi nel dialogo-racconto Amori sulla tela ${ }^{33}$ il 9 novembre 1986. Pertanto, la rivisitazione del volume di Doré durante l'assemblaggio della Ricerca è di fondamentale importanza: senza di esso, forse, Levi non sarebbe incappato nell'incisione della "ragna" e non avrebbe prodotto questo compatto e originale insieme di testi dedicati agli aracnidi. Le lenti di questo caleidoscopio emotivo saranno le stesse che si consolideranno nella meditazione sull'incisione di Doré, le stesse che lo accompagneranno nel corso di tutta la sua parabola letteraria, fino alla morte.

Resta infatti ancora un tassello da analizzare: i motivi affrontati finora sono tutti riassunti nell'ultima 'storia naturale' di Primo Levi, il quale, fino al suo testo più tardo, continuò a esercitare il suo occhio di naturalista, creando un canale di comunicazione tra la sua passione scientifica e le sue doti letterarie. Siamo nel 1986: se da un lato della sua carriera di scrittore offriva un risolutivo saggio di straordinaria potenza come I sommersi e $i$ salvati, dall'altra si impegnava ancora nella composizione di piccoli divertissement, brevi testi divertenti ma utili. La rivista "Airone", dall'aprile 1986 al maggio 1987, gli dedicò una rubrica, "Zoo immaginario. Le storie naturali di Primo Levi", che ospitò quell'originale serie di conversazioni immaginarie in cui il chimicoscrittore saggiava, con spirito, acume e rigore biologico, il mondo animale: ${ }^{34} \mathrm{si}$ tratta, come è stata definita, di una "serie di dialoghi umoristici e poesie scritti nell'ultimo periodo di Levi, in cui agli animali e ad altri organismi viene data una voce per narrare e descrivere le loro vite e il loro habitat" ${ }^{35}$. Gli aracnidi

31. Da ricordare è che, al di là della questione erotica analizzata in questa sede e della componente finzionale nei testi, era la ragnatela a destare in Levi lo stupore più forte: lo conferma l'articolo Il segreto del ragno, del 9 novembre 1986 (PS, II, pp. 1647-1649), in cui "lo scrittore passa da un argomento autobiografico a un tema chimico e scientifico, per chiudere con una riflessione naturalistica sui ragni ingegnosi" (Pagine sparse 1947-1987. Note ai testi, II, p. 1850). È proprio nel comprendere l'“industriosità del ragno” (Belpoliti, 2015, p. 247) che lascia uno dei suoi commenti più memorabili: parlando della ragnatela, osserva che "nessun chimico è ancora riuscito a riprodurre un procedimento così elegante, semplice e pulito. Abbiamo sorpassato e violentato la natura in molti campi, ma dalla natura abbiamo ancora parecchio da imparare" (PS, II, p. 1649).

32. Cfr. Note ai testi. La ricerca delle radici, II, p. 1785.

33. PS, II, pp. 1685-1687.

34. Rimando alla voce Ragno in Belpoliti, 1997, pp. 199-201, e anche al più generale lemmario sinottico dedicato agli animali nell'opera di Levi.

35. La definizione è di Cicioni, 2007, p. 145 (la traduzione italiana è mia), dove l'importanza 
ritornano in Amori sulla tela, dove Levi veste i panni di un giornalista che si reca presso una "signora ragna" 36 per intervistarla. Dopo averle chiesto di spiegare perché stia sempre a testa in giù ed averla interrogata sull'arte della tessitura, giunge al punto saliente e le domanda circa la riproduzione e i comportamenti di coppia:

Ora mi dica: corrono certe voci sul suo comportamento, diciamo così, matrimoniale ... solo voci, intendiamoci, io personalmente non ho mai visto niente di riprovevole, ma sa bene, la gente mormora ...

Lei vuole alludere al fatto che il maschio noi ce lo mangiamo? E tutto qui? Ma sicuro, ma certo. È una specie di balletto; i nostri maschi sono magrolini, timidi e deboli, neppure tanto bravi a farsi una tela come si deve. Quando sentono crescere il desiderio si avventurano sulle nostre tele, passo passo, incerti, esitanti, perché sanno anche loro come può andare a finire. Noi li aspettiamo: non prendiamo iniziative, il gioco è chiaro per tutte e due le parti. A noi femmine i maschi piacciono come le mosche se non di più. Ci piacciono in tutti i sensi della parola, come mariti (ma soltanto per il minimo tempo indispensabile) e come alimento. Una volta che hanno adempiuto alla loro funzione per noi perdono ogni attrattiva salvo quella della carne fresca, e così, in un colpo solo, ci riempiono lo stomaco e la matrice. ${ }^{37}$

Ritorna sulla scena il tema più evidente della poesia Aracne, a cui questo dialogo-racconto è collegato fuor di dubbio; e con esso, in filigrana, ecco venire anche quel recondito éros di cui, nella lettura di Levi, la raffigurazione di Doré era impregnata. I toni diventano qui molto più partecipati, più sciolti e colloquiali rispetto alle prime domande, invece più formali e contenute. Lo conferma la penultima battuta, prima dei saluti, in cui l'intervistatore si sbilancia in un'osservazione personale e leggermente irriverente, quando considera e commenta la soluzione di alcuni ragni maschi che, per evitare di essere mangiati, saziano la fame delle loro compagne offrendo loro prede preparate in precedenza:

Mi sembra un sistema ingegnoso, e tutto compreso rientra in una certa logica. Anchio, al loro posto, farei cosi, ma capisce, mia moglie ha meno appetito e un carattere più mite; e poi i nostri matrimoni durano a lungo, a noi sembrerebbe un peccato accontentarsi di una copula sola. ${ }^{38}$

Seppure i ragni siano "animali ambivalenti" in Levi, ${ }^{39}$ come ha giustamente puntualizzato Marco Belpoliti, non c'è da temere un giudizio negativo da parte del chimico-scrittore, che in Paura dei ragni rassicura i suoi lettori (e se stesso) rifacendosi al leopardiano Passero solitario: "l'animale non può essere oggetto di giudizi morali, 'ché di natura è frutto / ogni nostra vaghezza'; e tanto meno dovremmo essere tentati di esportare i nostri criteri morali umani ad animali

di questi scritti è messa in prospettiva con l'umorismo primoleviano.

36. PS, II, pp. 1685.

37. PS, II, p. 1686.

38. PS, II, p. 1687.

39. Belpoliti, 2015, p. 245. 
tanto lontani da noi come gli artropodi" ${ }^{40}$ Eppure, resta il fatto che i costumi sessuali degli aracnidi - sui quali insiste quando impersona Aracne, ai quali si interessa quando veste i panni dell'intervistatore - esercitavano su Levi un forte fascino, sin da Lo scrittore e gli animali: la tentazione di rivedersi in loro glieli faceva descrivere come esseri "pieni di un loro tenebroso significato, che destano risonanze sorde nel profondo delle nostre coscienze di civilizzati”. ${ }^{41}$ Una risonanza che, per lui, scattava ogni qualvolta vedesse un ragno, ${ }^{42} \mathrm{o}$ peggio una 'ragna': d'un tratto, si riaffacciava alla sua mente lo sguardo di Dante, rapito e completamente assorto dalla bestiale trasformazione, dalla forma ferina che Aracne stava assumendo. Dunque Levi fu davvero voyeur del voyeur, e tenne ben impressa in mente un'immagine che toccava nel vivo la sua pudicizia e il suo ribrezzo: per questo motivo è forse ancora possibile scorgere, al tornasole, quel curioso desiderio di comprensione innescato dall'illustrazione di Doré, nonché l'atto di nascita della personale e ossimorica attraente paura di Levi, grazie alla quale possiamo conoscere la sublime, vivida lettura che ha dato della raffigurazione di Aracne. ${ }^{43}$ Così come nella prassi ecfrastica hanno un ruolo preponderante le "passioni soggettive ed espansioni narrative" (per usare di nuovo le efficaci parole di Fusillo), ${ }^{44}$ anche nel tentativo di Levi possiamo ammirare una polimerizzazione di straordinaria potenza tra immagine e testo, tra oggettività e soggettività. Questo è il legame che unisce l'arte figurativa e la letteratura, indissolubili, longeve e feconde arti sorelle.

40. La citazione nella citazione proviene da AM, II, 908.

41. AM, II, p. 853

42. La sua aracnofobia non cessò mai, a quanto riferisce Thomson, 2002, p. 33.

43. Questo è il meccanismo di un rapporto intertestuale ed intersemiotico molto simile, definito da Rosati nel suo commento a Met. VI, e che potrebbe ritrovarsi anche nei testi di Levi dedicati ai ragni e ad Aracne: "L'ékphrasis, infatti, in quanto lettura e interpretazione di un prodotto artistico (e sua traduzione da un medium espressivo a un altro, quello poetico, che entra in competizione a sua volta), costituisce spesso l'occasione per una riflessione, un rispecchiamento che l'opera descritta riverbera su quella che la contiene al suo interno" (Ovidio, 2009, p. 245).

44. Così scrive Fusillo, 2014, p. 25, riepilogando le capacità umane di adattamento dei testi, specie se intendiamo questi ultimi secondo la felice metafora barthesiana di "tissu de citations, issues des mille foyers de la culture" (Barthes, 1984, p. 65), le cui trame si ricombinano e mescolano costantemente nell'“immaginario reticolare” di Domenichelli (vd. nota 14$)$. 


\section{Bibliografia}

Abignente, E. (2014). La letteratura e le altre arti, in F. De Cristofaro (Ed.). Letterature comparate (pp. 167-194). Roma: Carocci.

Alighieri, D. (1954). La Divina Commedia (con 100 illustrazioni di Gustave Doré). Milano: Mondadori.

Alighieri, D. (1868). La Divina Commedia illustrata da Gustavo Doré (a cura di E. Camerini, 3 voll.). Milano: Sonzogno.

Barthes, R. (1984). Le Bruissement de la langue. Essais critiques IV. Paris: Seuil.

Belpoliti, M. (1997). Animali, in M. Belpoliti (Ed.). Riga 13. Primo Levi (pp. 157 209). Milano: marcos y marcos.

Belpoliti, M. (2015). Primo Levi di fronte e di profilo. Milano: Guanda, PDF e-book.

Belpoliti, M. \& Gordon, R. S. C. (2007). Primo Levi's holocaust vocabularies, in R. S. C. Gordon (Ed.). The Cambridge Companion to Primo Levi (pp. 51-66). Cambridge: Cambridge University Press.

Bucciantini, M. (2016, luglio). Levi, una vita da fantascienza. IlSole24Ore. Disponibile su https://st.ilsole24ore.com/art/cultura/

Calcagno, G. (2000). Dante dolcissimo padre, in E. Mattioda (Ed.). Al di qua del bene e del male. La visione del mondo di Primo Levi (pp. 167-174). Milano: FrancoAngeli.

Cicioni, M. (2007). Primo Levi's humour, in R. S. C. Gordon (Ed.). The Cambridge Companion to Primo Levi (pp. 137-154). Cambridge: Cambridge University Press.

Cravero, M. (2020). Primo Levi e Ovidio. "Nel mondo delle cose che mutano": tra racconto metaforico e mito metamorfico, in G. Cinelli e R. S. C. Gordon (Edd.). Innesti. Primo Levi e i libri altrui (pp. 361-380). Bern: Peter Lang.

Domenichelli, M. (2008). L'immaginario reticolare: memoria personale, memoria culturale e tematologia, AllegoriaOnline, 58 (3), 34-48.

Fusillo, M. (2012). Feticci. Letteratura, cinema, arti visive. Bologna: il Mulino.

Fusillo, M. (2014) Passato presente futuro, in F. De Cristofaro (Ed.). Letterature comparate (pp. 13-31). Roma: Carocci.

Graves, R. (1983). I miti greci. Milano: Longanesi, PDF e-book.

Levi, P. (2016-2018). Opere complete (a cura di M. Belpoliti, introduzione di D. Del Giudice, 3 voll.). Torino: Einaudi.

Mila, M. (1997). Il sapiente con la chiave a stella, in M. Belpoliti (Ed.). Riga 13. Primo Levi (pp. 144-145). Milano: marcos y marcos.

Ovidio, P. N. (2009). Metamorfosi: vol. 3. Libri V-VI (a cura di G. Rosati, G. Chiarini trad.). Milano: Fondazione Lorenzo Valla - Mondadori.

Thomson, I. (2002). Primo Levi. A life. New York: Metropolitan Books.

Thomson, I. (2017). Primo Levi. Una vita. (E. Gallitelli trad.). Milano: Utet.

Usher, J. (2004). "Libertinage": Programmatic and Promiscuous Quotation in Primo Levi, in J. Farrell (Ed.). Primo Levi: The Austere Humanist (pp. 91-116). Bern: Peter Lang. 
